\title{
Variations in internal standard: an inspector's perspective
}

\author{
Stephen Vinter*,1 \\ ${ }^{1}$ MHRA, 10 South Colonnade, Canary Wharf, London, E14 4PU, UK \\ *Author for correspondence: stephen.vinter@mhra.gov.uk
}

\author{
"How a laboratory manages, documents and reports internal standard response review can \\ provide important information to inspectors about the data, systems and the level of compliance \\ of the laboratory being inspected."
}

First draft submitted: 19 September 2019; Accepted for publication: 30 September 2019; Published online: 16 October 2019

\author{
Keywords: bioanalysis $\bullet$ chromatography $\bullet$ good clinical practice $\bullet$ good laboratory practice $\bullet$ internal standard $\bullet$ \\ regulatory inspection
}

Inspections play a crucial part in the regulatory process ensuring studies are conducted in accordance with relevant regulations and allowing verification of the data generated by a laboratory. If these requirements are satisfied, then the assessment of data supplied to support regulatory decisions is a more straightforward process for both applicants and the regulator.

Medicines \& Healthcare products Regulatory Agency (London, UK) inspections cover several areas of laboratory activity to enable an assessment to be made of the facility's systems, its staff and the integrity of the data it has generated. Inspections will often include tours of the facility, interviews with staff, review of the Quality Management System, equipment reviews, data integrity checks and reconstruction of the data generation.

What part do internal standards and a review of their response play in an inspection? The answer to this question is highlighted throughout this special issue, but the short answer is that they play an important part in supporting the quality of the data by giving information related to the assay performance, so there is an expectation that a laboratory reviews this response and acts accordingly.

Inspectors continue to identify issues where an inconsistent internal standard response within an assay has not been reviewed by a laboratory, where current guidance has been incorrectly interpreted or where there is a lack of transparency in how the variation in response was assessed and mitigated. In some cases, this has resulted in rejection of data from the impacted studies.

How a laboratory manages, documents and reports internal standard response review can provide important information to inspectors about the data, systems and the level of compliance of the laboratory being inspected.

It is important to consider the underlying principles associated with regulatory studies in addition to specific requirements of guidance documents. If all these principles are incorporated into a laboratory's systems and culture successfully, then a significant step will have been taken in generating reliable and compliant data.

\section{Good laboratory practice \& good clinical practice}

The quality and validity of study data, plus the ability to verify study conduct, are important factors when we consider the principles of both good laboratory practice (GLP) and good clinical practice (GCP).

If we consider the Organisation for Economic Co-operation and Development (Paris, France) principles of GLP; the conduct of the study is the responsibility of the Study Director. When the Study Director signs and dates the final report, they are indicating acceptance of responsibility for the validity of the data [1]. In addition, a key principle of GCP can be found in the International Council for Harmonisation (ICH) E6 (2.13), which requires that systems with procedures that assure the quality of every aspect of the trial should be implemented [2].

An assessment of internal standard response can aid the Study Director while making the statement of compliance to confirm the validity of the data, which is a key statement used by regulators when assessing the compliance 
of a GLP study. The internal standard response assessment will also support the requirements discussed in ICH E6 to ensure the quality of every aspect of the trial. Therefore, poor internal standard response may undermine the validity and quality (reliability) of the data, so without making this assessment and appropriately assessing the impact, a study may not be compliant with the principles of GLP or GCP. An assessment of internal standard response is therefore considered necessary and should be effectively documented.

Contemporaneous and accurate recording of study events is extremely important when considering effective documentation. This recording of events and study decisions is extremely important when considering that an inspector will need to verify the study conduct. During GLP facility inspections, the quality and integrity of data generated by the facility are assessed and during study audits, an inspection will need to establish whether practices were employed in the development of data that would impair their validity [3]. ICH E6 (2.10) requires that all clinical trial information should be recorded, handled and stored in a way that allows its accurate reporting, interpretation and verification [2].

\section{What can we take from these principles regarding internal standard response?}

There are many components to an inspector's assessment of the study conduct and the validity of the data generated. It is reasonable to conclude that by assessing internal standard response, a laboratory has both a powerful tool for internal decision making and also valuable information to allow verification of the study activities and results. When suitable documentation, including the subsequent decisions, are combined with the assessment, an inspector will be able to use this information to support their inspection activities to verify the quality and validity of the reported data.

\section{Current guidance from regulators in Europe \& the USA}

Current regulatory guidance includes reference to internal standard response, so it is useful to pay attention to the content of these documents, especially if there is a requirement for an assay to comply with them.

The European Medicines Agency Guideline on Bioanalytical Method Validation [4] allows sample re-analysis if the internal standard response of the unknown is significantly different from the response of the calibration standard and quality control samples, when criteria for re-analysis have been predefined in a Standard Operating Procedure (SOP). The European Medicines Agency Reflection paper for laboratories that perform the analysis or evaluation of clinical trial samples [5], also reflects the ICH E6 principles that we have already discussed, in that in all cases sufficient documentation should be available to confirm that the conduct of the analysis is performed in a manner which assures its quality. The current US FDA Bioanalytical Method Validation guidance [6] also requires that internal standard response of calibration standards are monitored for variability and that a SOP is in place to address such issues.

So using these guidelines and reflection papers as examples and then adding the principles of GLP and GCP, plus any other local regulatory requirements, it is fairly clear that laboratories should have procedures in place to assess internal standard response to ensure the quality and validity of data and suitable documentation of the assessment to support decision making.

\section{Inspection experiences \& expectations}

This inspector's experience has seen well-established SOPs, good documentation and technically sound decision making which is fully transparent to allow straightforward verification of study conduct. Unfortunately, there are numerous examples where inspection findings have been raised and, in some cases, resulted in the rejection of data. Inspection findings have included no assessments being made at all, limited assessment where simple outlier tests are applied without taking into account significant trends in the internal standard response, no documentation retained to support decision making, inappropriate acceptance criteria within SOPs or misinterpretation of guidance leading to unjustified repeat analysis or re-injection of samples.

\section{When should this assessment take place?}

Obviously this should take place during sample analysis as decisions will be made on whether repeat analysis is required and the assessment will also provide information about the performance of the assay and potential analytical issues. It is also advisable to assess internal standard response during method validation as there have been examples observed on inspection of internal standard response issues not being detected during the validation 
of the method which have then also occurred during sample analysis, leading to lengthy analytical investigations, repeated validation experiments and lost data.

As an expectation, an inspector would expect to see a procedure in place (that is in compliance with regulatory requirements) for internal standard response assessment, which looks for outliers and assesses any trends in the response, which then triggers appropriate actions (such as investigations, repeat analysis or the acceptance of data). These decisions should be suitably documented to allow verification of the study conduct, to understand the rationale behind the decision and to provide evidence that the data are valid and of suitable quality. It is useful to also give some attention to final report transparency. Information regarding issues or investigations should be accurately and completely described so that the decision maker, whether it is another scientist or a regulator, has all the necessary information to enable them to understand the full conduct of the study.

If these are all in place, then an inspector will be able to review the study documentation effectively, discuss any decision making with the laboratory and avoid significant inspection findings and subsequent issues with gaining regulatory approval.

It should also not be forgotten that the quality of data are of high importance as there will be an animal, volunteer, patient or the environment impacted by the laboratory's results. These data can be used before any inspection or regulatory submission so there is a responsibility to ensure that the data are of suitable quality to avoid any harm to the study subjects.

Hopefully this issue will provide information on how best to meet the needs of regulators, laboratory best practice and things to look out for when considering the impact internal standard response can have on bioanalytical data.

By sharing our combined experiences on this issue, we can hopefully all understand the challenges that laboratories face in this technically demanding field and generate reliable and compliant data.

\section{Acknowledgments}

I would like to thank the Medicines \& Healthcare products Regulatory Agency laboratories inspection team for their ongoing support and input to enable the writing of this editorial.

\section{Financial \& competing interests disclosure}

The author has no relevant affiliations or financial involvement with any organization or entity with a financial interest in or financial conflict with the subject matter or materials discussed in the manuscript. This includes employment, consultancies, honoraria, stock ownership or options, expert testimony, grants or patents received or pending, or royalties.

No writing assistance was utilized in the production of this manuscript.

\section{Open Access}

This work is licensed under a Crown Copyright protection and licensed for use under the Open Government License unless otherwise indicated. Where any of the Crown copyright information in this work is republished or copied to others, the source of the material must be identified and the copyright status under the Open Government License acknowledged.

\section{References}

1. Organisation for Economic Co-operation and Development. Principles of good laboratory practice (1998). www. oecd.org/chemicalsafet y/testing/oecdseriesonprinciplesofgoodlaboratorypracticeglpandcompliancemonitoring.htm

2. International Council for Harmonisation. Guideline for good clinical practice E6(R2) (2017). www.ich.org/fileadmin/Public_Web_Site/ICH_Products/Guidelines/Efficacy/E6/E6_R2__Step_4_2016_1109.pdf

3. Organisation for Economic Co-operation and Development. Guidance for GLP monitoring authorities, revised guides for compliance monitoring procedures for good laboratory practice (1995). www.oecd.org/chemicalsafety/testing/oecdseriesonprinciplesof goodlaboratorypracticeglpandcompliancemonitoring.htm

4. European Medicines Agency. Guideline on bioanalytical method validation (2012). www.ema.europa.eu/en/documents/scientific-guideline/guideline-bioanalytical-method-validation_en.pdf

5. European Medicines Agency. Reflection paper for laboratories that perform the analysis or evaluation of clinical trial samples (2012). www.ema.europa.eu/en/documents/regulatory-procedural-guideline/reflection-paper-laboratories-perform-analysis-ev aluation-clinical-trial-samples_en.pdf

6. US FDA, Department of Health and Human Services, Center for Drug Evaluation and Research. Bioanalytical method validation guidance for industry (2018). www.fda.gov/media/70858/download 
